\title{
Lipid Composition in the Classification of the Butyric Acid-producing Clostridia
}

\author{
BY NORAH C. JOHNSTON AND HOWARD GOLDFINE* \\ Department of Microbiology, School of Medicine, University of Pennsylvania, Philadelphia, \\ Pennsylvania 19104, U.S.A.
}

(Received 22 July 1982; revised 16 September 1982)

\begin{abstract}
An examination of 20 strains of butyric acid-producing Clostridium species for phospholipid class compositions, plasmalogen content, and acyl and alk-1-enyl chains showed that the deoxyribonucleic acid homology groups I (Clostridium butyricum) and II (Clostridium beijerinckii) could be distinguished by their lipid compositions. The phospholipids of $C$. butyricum strains had ethanolamine as the major nitrogenous lipid polar head-group moiety, more octadecenoate plus $\mathrm{C}_{19}$-cyclopropane than hexadecenoate plus $\mathrm{C}_{17}$-cyclopropane acyl chains, and the predominant alk-1-enyl chain was $\mathrm{C}_{18}$-monounsaturated. Clostridium beijerinckii strains had $N$-methylethanolamine plus ethanolamine in phospholipid head-groups, more hexadecenoate plus $\mathrm{C}_{17}$-cyclopropane than octadecenoate plus $\mathrm{C}_{19}$-cyclopropane acyl chains, and the major alk-1-enyl chain was $\mathrm{C}_{16}$-saturated. Three species falling outside the two homology groups Clostridium fallax, Clostridium pseudofallax and Clostridium acetobutylicum had ethanolamine as the major phospholipid base, but these species could be distinguished from $C$. butyricum by their acyl and alk-1-enyl chain compositions. The lipid composition of Clostridium pasteurianum is even more distinct.
\end{abstract}

\section{INTRODUCTION}

On the basis of cell wall sugar compositions and DNA homologies, Cummins \& Johnson (1971) gathered many strains of butyric acid-producing clostridia into two DNA homology groups. Organisms in homology group I had cell walls containing glucose and grew in a mineral salts-glucose medium supplemented with biotin. Organisms in homology group II had both glucose and galactose in their cell walls and required supplementation with yeast extract in the presence of amino acids and ten vitamins for growth. These authors recommended that homology group I strains retain the name Clostridium butyricum, and that strains in homology group II be designated Clostridium beijerinckii. Some strains labelled Clostridium fallax and strains of Clostridium acetobutylicum, Clostridium aurantibutyricum, Clostridium pasteurianum, and Clostridium tyrobutyricum were not placed in these two homology groups.

In studies on the lipid compositions of butyric acid-producing clostridia designated $C$. butyricum, differences in lipid composition have been noted. For example, the predominant nitrogenous polar head-group moiety in C. butyricum ATCC 6015 was found to be $N$ methylethanolamine (Goldfine, 1962; Baumann et al., 1965), whereas that of $C$. butyricum IFO 3852 was found to be ethanolamine (Matsumoto et al., 1971). Both strains are rich in plasmalogens and contain an unusual glycerol acetal of the major plasmalogen (Matsumoto $e t$ al., 1971), which differs in its polar head-group base in the two strains as noted above. The proportion of this lipid has been found to increase in cells grown on certain unsaturated fatty acids in the absence of biotin (Khuller \& Goldfine, 1975; Goldfine et al., 1977), and its thermotropic phase behaviour has revealed an unusually large hysteresis of the liquid-crystalline 
to gel phase transition (Goldfine et al., 1981). We have, therefore, studied the distribution of ethanolamine and $\mathrm{N}$-methylethanolamine-containing phospholipids and the fatty acyl and alkl-enyl group compositions of the total phospholipids in a number of strains of butyric acidproducing clostridia. Our studies show that homology groups I and II of Cummins \& Johnson (1971) can be clearly distinguished on the basis of their lipid compositions in addition to their cell wall sugars and nutritional requirements.

\section{METHODS}

The sources and designations of the strains we have studied are given in Table 1. ATCC or VPI strains were first grown on chopped meat medium (Scot Labs, Fiskville, Rhode Island, U.S.A.) then transferred to peptone/yeast extract/glucose medium (Holdeman et al., 1977). Inocula for large cultures were also grown on peptone/yeast extract/glucose medium. The large cultures of cells $(2-41)$ for lipid analysis were grown on the medium described by Cummins \& Johnson (1971). Anaerobic conditions were maintained in tubes by cooling sterilized medium under $\mathrm{CO}_{2}$. The tubes were inoculated and traces of oxygen were removed by double plugging using alkaline pyrogallol (Hardman \& Stadtman, 1960). When the medium was prepared in flasks, the glucose, cysteine and sodium bicarbonate were sterilized separately and all solutions cooled under $\mathrm{CO}_{2}$ by blowing the gas into the neck of the flask through a sterile syringe needle; the solutions were added when cool, before inoculating with a $10 \%$ (v/v) inoculum. The flasks were sparged with $\mathrm{CO}_{2}$ before being incubated at $37^{\circ} \mathrm{C}$. Cells usually grew overnight and were harvested in late-exponential or stationary phase. The cell paste was washed twice with $0.05 \mathrm{M}$-potassium phosphate $\mathrm{pH} 7.2$ and either extracted immediately or frozen at $-20^{\circ} \mathrm{C}$.

Extraction, separation and analysis of lipids. The cell paste was suspended in chloroform/methanol $(2: 1, \mathrm{v} / \mathrm{v})$ and extracted for $1 \mathrm{~h}$ as described by Goldfine \& Bloch (1961). The crude lipid extract was chromatographed on silicic acid columns (Bio-Sil A, Bio-Rad); non-polar lipids and fatty acids were eluted with chloroform followed by acetone, and the phospholipids with chloroform/methanol $(1: 2, \mathrm{v} / \mathrm{v})$.

The intact phospholipids were analysed by TLC on silica gel G plates (Prekote, Applied Science, State College, Pa., U.S.A.) or on high-performance thin-layer (HPTLC) plates (Merck, no. 5631) using the following solvent systems: (a) chloroform/methanol/7 M-ammonium hydroxide (12:7:1, by vol.) (Skidmore \& Entenman, 1962); (b) $n$-butanol/acetic acid/water $(3: 1: 1$, by vol.) (Kates, 1972); (c) chloroform/methanol/acetic acid $(65: 25: 8$, by vol.). Sequential hydrolysis of the phospholipids began with acid hydrolysis. Lipid ( $2-5 \mathrm{mg}$ ) was treated with $90 \%$ $(\mathrm{v} / \mathrm{v})$ acetic acid $(\mathrm{l} \mathrm{ml})$ at $37^{\circ} \mathrm{C}$ overnight. After removal of acetic acid by lyophilization, the aldehydes, produced by hydrolysis of plasmalogens and glycerol acetals, and phospholipids were separated on small $(0 \cdot 2-0.5 \mathrm{~g})$ silicic acid columns. Chloroform $(4-5 \mathrm{ml})$ plus $1 \%(\mathrm{v} / \mathrm{v})$ methanol in chloroform $(1 \mathrm{ml})$ eluted the aldehydes and $5-6 \mathrm{ml}$ of chloroform/methanol $(1: 2, \mathrm{v} / \mathrm{v})$ eluted the diacyl- and lyso-phospholipids. The phospholipids recovered from acid hydrolysis were further hydrolysed by alkaline methanolysis (Kates, 1972). Glycerophosphate esters were analysed by two-dimensional TLC by the method of Short $e$ e al. (1969). The aldehyde and fatty acid methyl ester (FAME) fractions were purified when necessary by TLC on pre-washed silica gel (Merck silica gel $60 \mathrm{HR}$, no. 7744) plates in the systems hexane/chloroform/methanol (27:12:1, by vol.) (Gilbertson et al., 1967) for the aldehydes and petroleum ether/diethyl ether/acetic acid $(90: 10: 1$, by vol.) for the esters; the spots were scraped into glass-wool plugged Pasteur pipettes and eluted with chloroform (aldehydes) or diethyl ether (FAME). Reagents for detection of spots on chromatograms were: (a) the molybdate reagent of Dittmer \& Lester (1964) for all phosphatecontaining compounds; $(b)$ ninhydrin $(0.3 \%$ in ethanol) for ethanolamine compounds; $(c) 0.4 \%$ dinitrophenylhydrazine in $2 \mathrm{M}$-hydrochloric acid for plasmalogens and glycerol acetals; $(d)$ Rhodamine B $(0.03 \%$ in ethanol) (Skipski \& Barclay, 1969) for FAME; (e) plates were sprayed with water to detect aldehydes. The FAME and aldehyde fractions were analysed by GLC using a Perkin Elmer 990 instrument equipped with a hydrogen flame ionization detector. The column, $1.83 \mathrm{~m} \times 3.2 \mathrm{~mm}$ (o.d.), of $10 \%(\mathrm{w} / \mathrm{w})$ diethylene glycol succinate on $80 / 100$ Supelcoport (Supelco, Bellefonte, Pa., U.S.A.) was operated at $170{ }^{\circ} \mathrm{C}$ with $\mathrm{N}_{2}$ as carrier gas. The proportions of each component were determined by multiplying the peak height by the retention time (Kates, 1972). Phospholipid composition was determined as follows: after two-dimensional TLC in solvent systems $(a)$ and $(c)$, the spots were located by brief exposure to iodine vapour and scraped into acid-washed screw-capped glass tubes; lipid phosphorus was determined by the method of Bartlett (1959). The presence of the glycerol acetal of the plasmalogen form of phosphatidylethanolamine or the $\mathrm{N}$-methylethanolamine analogue was demonstrated using detection method $(c)$ by TLC in system $(a)$ of parallel lipid samples before and after acid hydrolysis. Vinyl ether content was determined by the method of Gottfried \& Rapport (1962), modified to give a final volume of $2.0 \mathrm{ml}$.

Nineteen strains of the butyric acid group of Clostridium previously studied by Cummins \& Johnson (1971) and C. butyricum IFO 3852 were examined for their phospholipid polar head- 


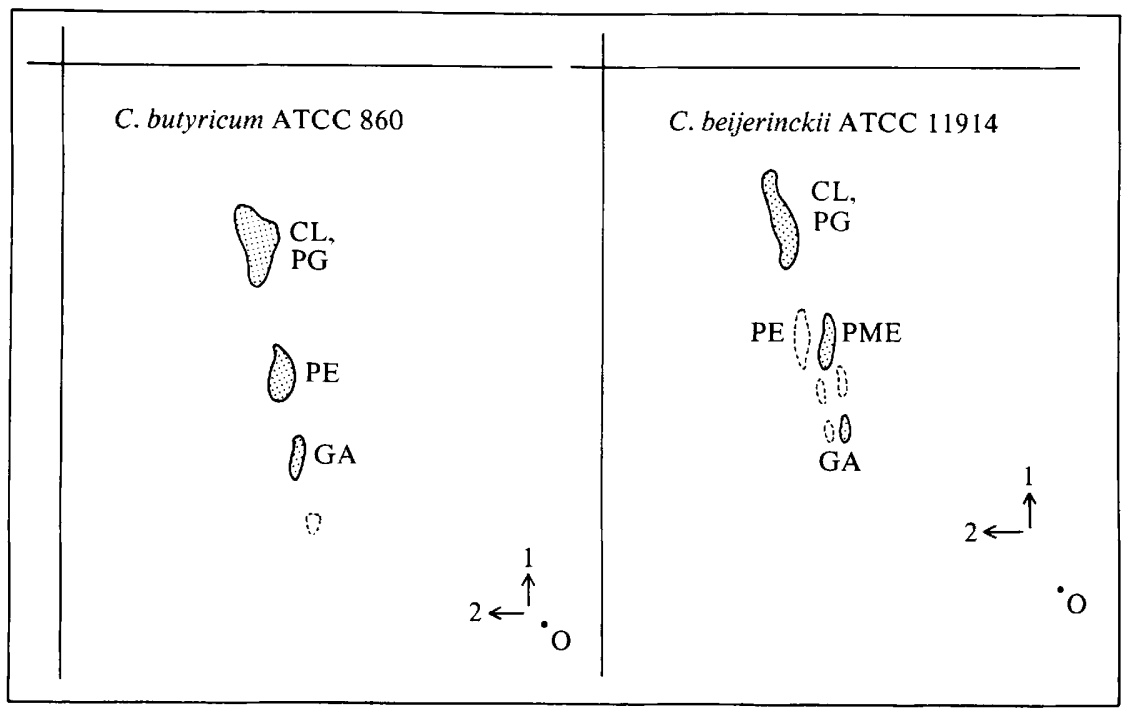

Fig. 1. Two-dimensional thin-layer chromatograms of phospholipids from Clostridium butyricum ATCC 860 and Clostridium beijerinckii ATCC 11914. Chloroform/methanol/7 $\mathrm{M}-\mathrm{NH}_{4} \mathrm{OH}(12: 7: 1$, by vol.) was used in the first dimension, and $n$-butanol/acetic acid/water $(3: 1: 1$, by vol.) in the second dimension. Abbreviations: CL, cardiolipin; PG, phosphatidylglycerol; PE, phosphatidylethanolamine; PME, phosphatidyl- $N$-methylethanolamine; GA, glycerol acetal lipids; O, origin.

groups both by TLC of the intact lipids (Fig. 1) and by TLC of the glycerophosphate esters obtained by sequential mild acid hydrolysis and alkaline methanolysis of the polar lipids. Analyses of the aldehydes obtained by mild acid hydrolysis of the alk-1-enyl and glycerol acetal bonds and of the fatty acid methyl esters, both obtained from the polar lipids, were also done. The results of these analyses are presented in Tables 1 to 3 .

All of the strains placed in homology group I according to their DNA homologies, cell wall sugars, and nutritional requirements (Cummins \& Johnson, 1971) are characterized by the presence of ethanolamine as the major nitrogenous base in the phospholipids and a ratio of $(16: 1+17: \mathrm{cyc})$ to $(18: 1+19: \mathrm{cyc})$ acyl chains below $1 \cdot 0$. Most of these strains had $18: 1$ as the predominant alk-1-enyl chain except for $C$. butyricum (multifermentans) VPI 2399, in which the sum of $18: 1$ plus $19:$ cyc was slightly higher than the $16: 0$ alk-1-enyl chain (Table 3 ). All strains in homology group II had both $N$-methylethanolamine and ethanolamine as phospholipid nitrogenous moieties, a ratio of $(16: 1+17:$ cyc $)$ to $(18: 1+19:$ cyc $)$ acyl chains greater than $1 \cdot 5$, and $16: 0$ as the major alk-1-enyl chain.

When vinyl ether was measured by an iodometric procedure, the molar ratio of vinyl ether to lipid phosphorus was lower in homology group I than in homology group II strains (Table 1). This assay does not measure the glycerol acetal lipid which represented from 5.9 to $29.2 \%$ of the lipid phosphorus (Table 4). We observed that when a strain was grown on the Casamino acid medium of Broquist \& Snell (1951), it contained a higher proportion of the glycerol acetal lipid than when it was grown on the richer medium used by Cummins \& Johnson (1971) (data not shown). Thus, the differences in glycerol acetal content seen in Table 4 were probably more related to the media than to the strains studied. We have found the glycerol acetal class of lipid in all of the butyric acid-producing clostridia we have studied, with the exception of $C$. pasteurianum ATCC 6013, which has a lipid composition very different from those seen in other clostridia of this group (A. Kaufman and $\mathbf{H}$. Goldfine, unpublished observations).

With the exception of 17 :cyc acyl and alk-1-enyl chains, which were not found or were seen in only small amounts in homology group I strains, and some longer chain alkenyl and acyl groups seen in strains of both groups, the major components of the alkenyl and acyl chains were qualitatively alike (Tables 2 and 3). Both groups had high proportions of $16: 0$ acyl chains and in 
Table 1. Principal phospholipid nitrogenous base moieties, major alkenyl chains, unsaturated acyl chains, and vinyl ether contents of butyric acid-producing clostridia

Strain and number

Homology group I

C. butyricum IFO $3852 \ddagger$

C. butyricum ATCC $860 \S$

C. butyricum ATCC $19398 \S$

C. butyricum (multifermentans) VPI 2399||

C. butyricum (fallax) VPI 5982|

C. butyricum VPI 3005

C. butyricum (multifermentans) VPI 2402|

C. butyricum VPI 2419

Homology group II

C. butyricum ATCC 6015

C. beijerinckii ATCC $11914 \S$

C. beijerinckii ATCC $14949 \S$

C. beijerinckii (butyricum) VPI 2983|

C. beijerinckii (amylolyticum) VPI 2697|

C. beijerinckii (multifermentans) VPI 2408

C. beijerinckii ATCC 6014

C. beijerinckii (butyricum) VPI 2793||

Other strains in the 'butyric

acid group'

C. fallax ATCC 19400

C. pseudofallax VPI 2410

C. acetobutylicum ATCC 824

C. pasteurianum ATCC 6013

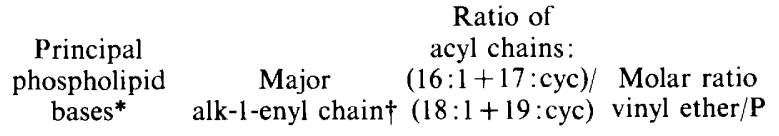

Etn
Etn
Etn
Etn
Etn
Etn
Etn
Etn

$18: 1$

$18: 1$

$0 \cdot 46$

$18: 1$

$0 \cdot 34$

$18: 1+19:$ cyc $\quad 0.43$

$18: 1 \quad 0.48$

$18: 1 \quad 0.59$

$18: 1 \quad 0.61$

$18: 1 \quad 0.67$

$0 \cdot 17$

$0 \cdot 16$

MeEtn, Etn 16:0 $\quad 5.5$

MeEtn, Etn 16:0 3.5

MeEtn, Etn 16:0 3.1

MeEtn, Etn 16:0 2.8

MeEtn, Etn 16:0 $\quad 5 \cdot 1$

$\begin{array}{lll}\text { MeEtn, Etn 16:0 } & 1.7\end{array}$

MeEtn, Etn 16:0 3.1

MeEtn, Etn $16: 0$

$6 \cdot 1$

$0 \cdot 34$

$0 \cdot 55$

$\begin{array}{llll}\text { Etn } & 16: 0 & 1 \cdot 1 & 0 \cdot 12 \\ \text { Etn } & 16: 0 & 0 \cdot 89 & 0 \cdot 16 \\ \text { Etn } & 19: \text { cyc } & 0 \cdot 37 & 0 \cdot 67 \\ & 17: \text { cyc } & \infty & \end{array}$

* Etn, ethanolamine; MeEtn, $N$-methylethanolamine.

+ The number before the colon is the chain length, the number after is the number of double bonds; cyc, cyclopropane.

$¥$ IFO 3852 is listed in homology group I based on its lipid composition. No studies on its DNA homology have been done.

$\S$ These acyl chain compositions represent only the diacyl phospholipids. The plasmalogen and glycerol acetal lipid acyl chains were analysed separately. The latter are both qualitatively and quantitatively similar to the acyl chains of the diacyl lipids and, since they represent about one-fourth of the total acyl chains in $C$. beijerinckii strains and one-eighth in $C$. butyricum strains, the approximate weighted average composition calculated is nearly indistinguishable from those presented.

|| Earlier designations listed by Cummins \& Johnson (1971). ATCC, American Type Culture Collection, Rockville, Md., U.S.A.; IFO, Institute of Fermentation, Osaka, Japan; VPI, Anaerobe Laboratory, Virginia Polytechnic Institute and State University, Blacksburg, Va., U.S.A.

homology group II $16: 0$ was the major alk-1-enyl chain. The high $(16: 1+17:$ cyc $)$ to $(18: 1+$ 19 : cyc) ratio seen in the acyl chains of homology group II strains, was also seen in the alk-1-enyl chains of all strains in this group, with the exception of VPI 2408 . The longer $18: 1$ chains were predominant among the unsaturated alk-1-enyl chains of strains in homology group I. Three of the strains not in homology groups I and II had ethanolamine, but no $N$-methylethanolamine in their phospholipids. Clostridium pasteurianum ATCC 6013 had no or very small amounts of phosphatidylethanolamine. The acyl chains of C. fallax ATCC 19400 and C. pseudofallax ATCC 2410 are intermediate in composition between homology groups I and II (Table 2). Palmitic acid was the predominant species, but the ratios of $(16: 1+17: \mathrm{cyc})$ to $(18: 1+$ $19: \mathrm{cyc})$ fell between those of groups I and II. The alkenyl chains were predominantly $16: 0$ in both strains (Table 3). Clostridium acetobutylicum was also characterized by a high proportion of $16: 0$ acyl chains, but $18: 1+19:$ cyc were the predominant alk-1-enyl chains.

Cummins \& Johnson (1971) showed that these latter species had low degrees of homology with representative $C$. butyricum and $C$. beijerinckii strains and that $C$. pasteurianum strains had very low DNA homologies. The lipid composition of $C$. pasteurianum ATCC 6013 was indeed 
Table 2. Acyl chain compositions of butyric acid-producing clostridia

Strain and number

Homology group I

C. butyricum IFO 3852

C. butyricum ATCC 860

C. butyricum ATCC 19398

C. butvricum VPI 2399

C. butyricum VPI 5982

C. butyricum VPI 3005

C. butyricum VPI 2402

C. butyricum VPI 2419

Homology group II

C. butyricum ATCC 6015

C. beijerinckii ATCC $11914 \dagger$

C. beijerinckii ATCC $14949 \dagger$

C. beijerinckii VPI 2983

C. beijerinckii VPI 2697

C. beijerinckii VPI $2408+$

C. beijerinckii ATCC 6014

C. beijerinckii VPI 2793

Other strains in the 'butyric acid group'
C. fallax ATCC 19400
C. pseudofallax VPI 2410
C. acetobutylicum ATCC 824
C. pasteurianum ATCC 6013

Acyl chain composition $(\%, w / w)^{*}$

\begin{tabular}{|c|c|c|c|c|c|c|c|}
\hline $14: 0$ & $14: 1$ & $16: 0$ & $16: 1$ & $17: \mathrm{cyc}$ & $18: 0$ & $18: 1$ & $19:$ cyc \\
\hline $14 \cdot 8$ & - & $43 \cdot 2$ & $13 \cdot 0$ & - & $2 \cdot 8$ & $24 \cdot 4$ & $3 \cdot 6$ \\
\hline $7 \cdot 2$ & - & $42 \cdot 4$ & $12 \cdot 1$ & - & $2 \cdot 5$ & $23 \cdot 2$ & $12 \cdot 5$ \\
\hline $5 \cdot 1$ & - & $47 \cdot 5$ & $8 \cdot 6$ & - & $4 \cdot 8$ & 18.9 & $14 \cdot 2$ \\
\hline $10 \cdot 0$ & $\operatorname{tr}$ & $48 \cdot 8$ & $11 \cdot 3$ & $\operatorname{tr}$ & $3 \cdot 2$ & 11.9 & $14 \cdot 3$ \\
\hline $10 \cdot 7$ & $\operatorname{tr}$ & $46 \cdot 7$ & $13 \cdot 2$ & - & 1.9 & $17 \cdot 5$ & $9 \cdot 8$ \\
\hline $13 \cdot 1$ & $\operatorname{tr}$ & $35 \cdot 2$ & $18 \cdot 1$ & $\operatorname{tr}$ & $1 \cdot 1$ & $21 \cdot 7$ & $9 \cdot 2$ \\
\hline $11 \cdot 2$ & $\operatorname{tr}$ & $40 \cdot 2$ & $18 \cdot 1$ & - & $1 \cdot 5$ & $28 \cdot 4$ & $1 \cdot 1$ \\
\hline $16 \cdot 5$ & $0 \cdot 5$ & $33 \cdot 0$ & $19 \cdot 6$ & 一 & 0.6 & $27 \cdot 2$ & $2 \cdot 2$ \\
\hline $3 \cdot 9$ & $0 \cdot 5$ & $58 \cdot 1$ & $25 \cdot 7$ & $5 \cdot 6$ & $\operatorname{tr}$ & $4 \cdot 6$ & $1 \cdot 1$ \\
\hline $4 \cdot 1$ & $\operatorname{tr}$ & $52 \cdot 05$ & $20 \cdot 8$ & $12 \cdot 2$ & $1 \cdot 4$ & 3.6 & $5 \cdot 9$ \\
\hline $2 \cdot 73$ & $0 \cdot 5$ & $50 \cdot 2$ & $25 \cdot 3$ & $7 \cdot 9$ & $1 \cdot 5$ & $7 \cdot 3$ & $3 \cdot 4$ \\
\hline $4 \cdot 14$ & $\operatorname{tr}$ & $60 \cdot 9$ & $12 \cdot 1$ & 11.9 & $2 \cdot 2$ & 1.9 & $6 \cdot 6$ \\
\hline $5 \cdot 5$ & $\operatorname{tr}$ & $54 \cdot 0$ & $15 \cdot 9$ & $17 \cdot 7$ & $1 \cdot 0$ & 1.64 & $4 \cdot 9$ \\
\hline $7 \cdot 6$ & $6 \cdot 4$ & $35 \cdot 5$ & $9 \cdot 6$ & $10 \cdot 1$ & $8 \cdot 3$ & $2 \cdot 3$ & $9 \cdot 58$ \\
\hline 3.9 & $0 \cdot 8$ & $48 \cdot 3$ & $28 \cdot 0$ & $6 \cdot 4$ & $1 \cdot 25$ & $8 \cdot 2$ & 3.03 \\
\hline $4 \cdot 5$ & 0.7 & $52 \cdot 6$ & $27 \cdot 5$ & $8 \cdot 5$ & - & $4 \cdot 4$ & $1 \cdot 5$ \\
\hline $7 \cdot 5$ & $1 \cdot 55$ & $45 \cdot 5$ & $20 \cdot 1$ & $2 \cdot 12$ & $1 \cdot 5$ & $17 \cdot 1$ & $3 \cdot 0$ \\
\hline $6 \cdot 4$ & 0.8 & $51 \cdot 4$ & $17 \cdot 8$ & 0.7 & $1 \cdot 8$ & $19 \cdot 8$ & 0.9 \\
\hline $8 \cdot 1$ & $\operatorname{tr}$ & $64 \cdot 5$ & $5 \cdot 6$ & $1 \cdot 0$ & 2.9 & $8 \cdot 8$ & $8 \cdot 8$ \\
\hline - & - & $92 \cdot 7$ & $3 \cdot 3$ & $4 \cdot 0$ & - & - & - \\
\hline
\end{tabular}

* Each value represents the mean of at least two analyses of a single sample of lipid from each strain; - , not detectable; $t r$, trace.

+ See footnote $\S$, Table 1 .

$\ddagger$ This strain also had $9 \cdot 8 \% 20: 0$.

Table 3. Alk-1-enyl chain compositions of butyric acid-producing clostridia

\begin{tabular}{|c|c|c|c|c|c|c|c|c|c|}
\hline \multirow[b]{2}{*}{ Strain and number } & \multicolumn{9}{|c|}{ Alk-1-enyl chain composition $(\%, w / w)^{*}$} \\
\hline & $14: 0$ & $14: 1$ & $16: 0$ & $16: 1$ & $17:$ cyc & $18: 0$ & $18: 1$ & $19:$ cyc & Unknown \\
\hline \multicolumn{10}{|l|}{ Iomology group I } \\
\hline butyricum IFO 3852 & $0 \cdot 3$ & - & $20 \cdot 3$ & $24 \cdot 7$ & - & $4 \cdot 6$ & $44 \cdot 6$ & $5 \cdot 3$ & \\
\hline butyricum ATCC 860 & $\operatorname{tr}$ & - & $23 \cdot 1$ & $22 \cdot 4$ & $1 \cdot 88$ & $3 \cdot 85$ & $40 \cdot 3$ & $8 \cdot 6$ & \\
\hline butyricum ATCC 19398 & $<1$ & - & $22 \cdot 9$ & $21 \cdot 1$ & $\operatorname{tr}$ & $4 \cdot 6$ & $43 \cdot 6$ & $7 \cdot 1$ & \\
\hline butyricum VPI 2399 & $1 \cdot 8$ & - & $32 \cdot 3$ & $14 \cdot 7$ & - & $7 \cdot 5$ & $24 \cdot 4$ & $9 \cdot 6$ & $5 \cdot 25,4 \cdot 25$ \\
\hline butyricum VPI 5982 & - & - & $13 \cdot 0$ & $14 \cdot 35$ & - & $5 \cdot 4$ & $49 \cdot 3$ & 5.9 & $11 \cdot 9$ \\
\hline butyricum VPI 3005 & $0 \cdot 6$ & $\operatorname{tr}$ & $8 \cdot 8$ & $15 \cdot 25$ & 0.45 & $5 \cdot 5$ & $44 \cdot 4$ & $3 \cdot 2$ & $17 \cdot 4,3 \cdot 4$ \\
\hline butyricum VPI 2402 & $0 \cdot 9$ & - & $12 \cdot 7$ & $19 \cdot 4$ & - & $4 \cdot 8$ & $58 \cdot 4$ & $3 \cdot 6$ & \\
\hline butyricum VPI 2419 & $\operatorname{tr}$ & $\operatorname{tr}$ & $10 \cdot 0$ & 35.9 & - & $2 \cdot 2$ & $49 \cdot 4$ & $3 \cdot 7$ & \\
\hline \multicolumn{10}{|l|}{ Iomology group II } \\
\hline butyricum ATCC 6015 & 0.55 & $\operatorname{tr}$ & $42 \cdot 6$ & $30 \cdot 6$ & $12 \cdot 5$ & $2 \cdot 5$ & $7 \cdot 8$ & $3 \cdot 3$ & \\
\hline beijerinckii ATCC 11914 & $\operatorname{tr}$ & - & $51 \cdot 4$ & $14 \cdot 0$ & $21 \cdot 1$ & $2 \cdot 4$ & $2 \cdot 05$ & 58.9 & \\
\hline beijerinckii ATCC 14949 & 0.5 & - & $43 \cdot 9$ & $20 \cdot 5$ & $21 \cdot 05$ & - & $5 \cdot 9$ & $8 \cdot 0$ & \\
\hline beijerinckii VPI 2983 & $0 \cdot 5$ & - & $55 \cdot 6$ & 6.6 & $18 \cdot 5$ & $6 \cdot 0$ & $2 \cdot 3$ & $10 \cdot 3$ & \\
\hline beijerinckii VPI 2697 & $0 \cdot 4$ & - & $68 \cdot 8$ & 4.9 & $15 \cdot 7$ & $4 \cdot 2$ & $\operatorname{tr}$ & $5 \cdot 6$ & \\
\hline beijerinckii VPI 2408 & $0 \cdot 4$ & - & $57 \cdot 4$ & $1 \cdot 76$ & $9 \cdot 4$ & $14 \cdot 6$ & $1 \cdot 18$ & $15 \cdot 1$ & \\
\hline beijerinckii ATCC 6014 & $0 \cdot 4$ & - & $45 \cdot 2$ & $27 \cdot 7$ & $9 \cdot 2$ & $3 \cdot 5$ & $9 \cdot 2$ & $4 \cdot 7$ & \\
\hline beijerinckii VPI 2793 & $0 \cdot 5$ & - & $52 \cdot 9$ & $22 \cdot 6$ & $15 \cdot 3$ & $\operatorname{tr}$ & $4 \cdot 6$ & 3.9 & \\
\hline \multicolumn{10}{|c|}{ ther strains in the "butyric acid group } \\
\hline fallax ATCC 19400 & 0.75 & $\operatorname{tr}$ & $96 \cdot 5$ & $2 \cdot 7$ & - & $\operatorname{tr}$ & $\operatorname{tr}$ & - & \\
\hline pseudofallax VPI 2410 & $2 \cdot 8$ & - & $68 \cdot 8$ & $15 \cdot 4$ & - & $5 \cdot 2$ & $7 \cdot 6$ & - & \\
\hline acetobutylicum ATCC 824 & $2 \cdot 3$ & $\operatorname{tr}$ & $17 \cdot 5$ & $7 \cdot 7$ & $5 \cdot 0$ & $5 \cdot 7$ & $15 \cdot 3$ & $46 \cdot 2$ & \\
\hline pasteurianum ATCC 6013 & - & - & $23 \cdot 5$ & $3 \cdot 0$ & $73 \cdot 6$ & 一 & - & - & \\
\hline
\end{tabular}

* Each value represents the mean of at least two analyses of a single sample of lipid from each strain; -, not detectable; tr, trace. 
Table 4. Phospholipid compositions of selected strains

\begin{tabular}{|c|c|c|c|c|c|}
\hline Strain and number & $\mathrm{PE}^{*}$ & PME* & $\begin{array}{l}\text { Glycerol } \\
\text { acetal lipid }\end{array}$ & $\mathrm{PG}^{*}$ & $\mathrm{CL}^{*} \dagger$ \\
\hline $\begin{array}{l}\text { Homology group I } \\
\text { C. butyricum ATCC } 19398 \ddagger \\
\text { C. butyricum IFO } 3852 \S\end{array}$ & $\begin{array}{l}56 \cdot 7 \\
39\end{array}$ & $\begin{array}{l}0 \\
0\end{array}$ & $\begin{array}{r}9 \cdot 1 \\
22 \cdot 6\end{array}$ & $\begin{array}{l}16 \cdot 3 \\
24 \cdot 8\end{array}$ & $\begin{array}{r}17.6 \\
8.9\end{array}$ \\
\hline $\begin{array}{l}\text { Homology group II } \\
\text { C. beijerinckii ATCC } 11914 \ddagger \\
\text { C. butyricum ATCC } 6015 \S\end{array}$ & \multicolumn{2}{|c|}{$47 \cdot 3 \|$} & $\begin{array}{r}5.9 \\
29.2\end{array}$ & $21 \cdot 5$ & $21 \cdot 7$ \\
\hline
\end{tabular}

* PE, phosphatidylethanolamine; PME, phosphatidyl- $N$-methylethanolamine; PG, phosphatidylglycerol; CL, cardiolipin. All of these lipid classes contain plasmalogens (Baumann et al., 1965; Matsumoto et al., 1971).

$\dagger$ Includes a polyglycerol phosphatide tentatively identified as acyl phosphatidylglycerol (Y. Koga \& H. Goldfine, unpublished observations).

† Grown on medium described by Cummins \& Johnson (1971).

$\S$ Grown on Casamino acid medium of Broquist \& Snell (1951). Data for C. butyricum ATCC 6015 from Baumann et al. (1965) and Goldfine et al. (1977).

"| Mainly PME (see Fig. 1).

very different from that of other organisms in the 'butyric acid group'. It had 92.7\% 16:0 acyl chains and $73.6 \% 17$ :cyc alk-1-enyl chains, but the structures of the polar lipids were not identified. On the basis of $23 S$ rRNA homologies, Johnson \& Francis (1975) placed C. butyricum and $C$. pasteurianum in separate subgroups of a very large rRNA homology group.

Clostridium butyricum IFO 3852, originally studied for lipid content by Matsumoto et al. (1971) represents an enigma. It is stated to be derived from $C$. butyricum ATCC 6014. However, ATCC 6014 is clearly a member of homology group II (Cummins \& Johnson, 1971) and IFO 3852 closely resembles homology group I strains in its lipid composition. Based on its lipid composition and other biochemical tests, strain IFO 3852 can no longer be considered to be a subculture of ATCC 6014 and has been discarded from the collection (Dr K. Imai, Curator, Institute for Fermentation, Osaka, personal communication).

The DNA homology and cell wall sugar data of Cummins \& Johnson (1971), together with the lipid composition presented here, suggest that the designation of C. butyricum ATCC 6015 should now be changed to $C$. beijerinckii, as was done earlier for other strains. Based on a review of its biochemical properties, the American Type Culture Collection will redesignate strain ATCC 6015 as C. beijerinckii (P. A. Pienta, Curator, ATCC, personal communication).

We are grateful to Dr W. E. C. Moore for strains and to Dr John C. McKitrick for helpful discussions. This research was supported by a Public Health Service research grant AI-08903 from the National Institute of Allergy and Infectious Diseases.

\section{REFERENCES}

Bartlett, G. R. (1959). Phosphorus assay in column chromatography. Journal of Biological Chemistry 234, 466-468.

Baumann, N. A., Hagen, P-O. \& Goldfine, H. (1965). Phospholipids of Clostridium butyricum. Studies on plasmalogen composition and biosynthesis. Journal of Biological Chemistry 240, 1559-1567.

Broquist, H. P. \& SNell, E. E. (1951). Biotin and bacterial growth. I. Relation to aspartate, oleate, and carbon dioxide. Journal of Biological Chemistry 188, 431-444.

Cummins, C. S. \& Johnson, J. L. (1971). Taxonomy of the clostridia: wall composition and DNA homologies in Clostridium butyricum and other butyric acidproducing clostridia. Journal of General Microbiology 67, 33-46.
Dittmer, J. C. \& Lester, R. L. (1964). A simple, specific spray for the detection of phospholipids on thin-layer chromatograms. Journal of Lipid Research 5, 126-127.

Gilbertson, J. R., Ferrell, W. J. \& Gelman, R. A. (1967). Isolation and analysis of free fatty aldehydes from rat, dog, and bovine heart muscle. Journal of Lipid Research 8, 38-45.

GoldFINE, H. (1962). The characterization and biosynthesis of an $\mathrm{N}$-methylethanolamine phospholipid from Clostridium butyricum. Biochimica et biophysica acta 59, 504-506.

GoldFine, H. \& BLOCH, K. (1961). On the origin of unsaturated fatty acids in clostridia. Journal of Biological Chemistry 236, 2596-2601.

Goldfine, H., Khuller, G. K., Borie, R. P., 
Silverman, B., Selick, H., Johnston, N. C., VANDERKOOI, J. M. \& HoRwITZ, A. F. (1977). Effects of growth temperature and supplementation with exogenous fatty acids on some physical properties of Clostridium butyricum phospholipids. Biochimica et biophysica acta 488, 341-352.

Goldfine, H., Johnston, N. C. \& Phillips, M. C. (1981). Phase behavior of ether lipids from Clostridium butyricum. Biochemistry 20, 2908-2916.

GotTFRIED, E. L. \& RAPPORT, M. M. (1962). The biochemistry of plasmalogens. I. Isolation and characterization of phosphatidal choline, a pure native plasmalogen. Journal of Biological Chemistry 242, 5700-5708.

Hardman, J. K. \& Stadtman, T. C. (1960). Metabolism of $\omega$-amino acids. I. Fermentation of $\gamma$ aminobutyric acid by Clostridium aminobutyricum $\mathrm{N}$. Sp. Journal of Bacteriology 79, 544-548.

Holdeman, L. V., Cato, E. P. \& Moore, W. E. C. (editors) (1977). Anaerobe Laboratory Manual, 4th edn. Blacksburg, Virginia: Virginia Polytechnic Institute and State University.

Johnson, J. L. \& Francis, B. S. (1975). Taxonomy of the clostridia: ribosomal ribonucleic acid homolo- gies among the species. Journal of General Microbiology 88, 229-244.

KATES, M. (1972). Techniques of lipidology. Isolation, analysis and identification of lipids. Amsterdam: North Holland Publishing Co.

K HUller, G. K. \& GoldFine, H. (1975). Replacement of acyl and alk-1-enyl groups in Clostridium butyricum phospholipids by exogenous fatty acids. Biochemistry 14, 3642-3647.

Matsumoto, M., Tamiya, K. \& Koizumi, K. (1971). Studies on neutral lipids and a new type of aldehydogenic ethanolamine phospholipid in Clostridium butyricum. Journal of Biochemistry 69, 617620.

Short, S. A., White, D. C. \& Aleem, M. I. H. (1969). Phospholipid metabolism in Ferrobacillus ferrooxidans. Journal of Bacteriology 99, 142-150.

Skidmore, W. D. \& Entenman, C. (1962). Twodimensional thin-layer chromatography of rat liver phosphatides. Journal of Lipid Research 3, 471 475.

Skipski, V. P. \& BaRClay, M. (1969). Thin-layer chromatography of lipids. Methods in Enzymology 14, 530-598. 